\title{
Introduction to the Health Information Behavior and Activity Tracking Technology Minitrack
}

\author{
Aylin Ilhan \\ Heinrich Heine University Düsseldorf \\ aylin.ilhan@hhu.de
}

\author{
Yuanyuan Feng \\ Carnegie Mellon University \\ yuanyuanfeng@cmu.edu
}

\section{Background}

Today, various consumer-facing wearable activity trackers (e.g., Fitbit, Apple Watch) provide a range of health-related functionalities, starting with counting steps, tracking sleep, monitoring heart rates and ending with evaluating exercise performance or providing personalized insights through data analytics. We use the term activity tracking technology to refer to the totality of activity trackers and related web or mobile health applications. The increasing ubiquity of this technology enables users to quantify and monitor their daily physical activities as part of their overall health, anytime and anywhere.

Researchers in various disciplines (e.g. computer science, information science, system sciences, engineering, medicine, and social sciences) have investigated different aspects regarding activity tracking technology, such as understanding the adoption and abandonment of activity trackers, optimizing accuracy of activity tracking technology, and supporting behavior change with this technology. For instance, Ilhan and Henkel [1] investigated the perceived service quality and acceptance of activity tracking users. Also, Feng and Agosto [2] has examined activity tracker users' personal health information practices with regard to this new technology.

Apart from that, it is still not well studied how people perceive, interact and make use of the manifold information offered by their activity trackers and related health applications. This evokes critical questions which needed to be examined to evaluate the impact of activity tracking technology on users' health information behavior. Some of the key questions are: Is the information provided by activity tracking technology adequate to satisfy users' various health information needs? How effective is the information offered by the technology in supporting users' desired behavioral change? What are the privacy implications as the wearable activity trackers become increasingly popular? What concerns do users' have if others (e.g. employers, insurance companies) have access to the information generated by the technology? How can we design privacy-enhancing wearable technology to safeguard activity tracker users' privacy related to their health information?

This minitrack aims to foster a wider and deeper discussion on health information behavior and activity tracking technology by exploring a subset of these critical questions.

\section{Introduction of papers}

\subsection{Workplace Health Promotion: Effects of an mHealth Application on Employee Behaviour and Wellness}

The promotion of health and including the support of increased physical activity is not only crucial for people regarding their daily life but also crucial in the workplace. Sedentary behavior is increasingly common in today's workplace, and the World Health Organization (WHO) has been supporting promotion for workplace health initiatives [3]. Health promotion initiatives not only cover physical activity but, for example, also include healthy eating. Our first presentation ties in with this topic.

Sophie Emerson, Ciara Heavin and Daniel J. Power investigated the impact of using a mHealth application in a workplace, considering internal motivation and external motivational aspects during a five-week challenge in Ireland. To answer if the mHealth application has a positive effect on employees, they used a mixed-methods approach. Among collecting quantitative data through the mHealth application, they conducted an online survey with employees who participated in the intervention and interviews as well. Apart from other findings, they stress that the design of an mobile activity-based tracking application should have an attractive interface. Their results also showed that the gamification element leaderboard was a popular and welcomed feature.

Apart from integrating activity tracking technologies 
in the workplace domain in the last few years, especially regarding misuse and security of data protection plays more and more an important role. Therefore, the next two papers investigating activity tracking technologies and privacy.

\subsection{Health Information, Human Factors and Privacy Issues in Mobile Health Applications}

With today's advanced technology, users have more and more concerns regarding their data protection. Notably, in the domain of health or fitness-related data, these data are sensitive for many users. Wagner Silva, Carolina Sacramento, Edenildo Silva, Ana Cristina, and Simone Bacellar point out that most research in the domain of privacy and security regarding activity tracking technologies is focusing on methods and techniques at the technical level. Therefore, they investigated privacy issues in mobile health application by focusing on the user's point of view.

To better understand the current research on user concerns about data privacy regarding mHealth applications, the authors conducted a systematic review of literature in the domain of Computer Science published between 2012 and 2018. They searched major literature databases for English papers published in journals, conferences, and book chapters. After screening 505 documents for scope and availability, they chose 21 papers for in-depth content analysis. The authors investigated, among others, the threats to privacy, including limited access permissions and improper information disclosure. Apart from the threads to privacy, users' demands are essential themes as well. The authors found out that most articles confirm users' demand to share information. The willingness and desire exist, but fears and concerns are preventing it.

This investigation has tackled the user needs to counteract the concerns and fears, which naturally leads to the third and last presentation that reveals more findings of activity tracking application users and their concerns and evaluation regarding the sensitivity of data.

\subsection{Fitness Tracking Technologies: Data Privacy Doesn't Matter? The (Un)Concerns of Users, Former Users, and Non-Users}

The authors Kaja J. Fietkiewicz and Aylin Ilhan draw on previous research investigating users' concerns about activity tracking technologies. They focused on users from the European Union and the USA. To find out the concerns of users and what kind of data they evaluate as sensitive, they conducted an online survey with 590 participants. These participants are characterized as a former user, a user, and a non-user of activity tracking technologies. Interestingly, the authors characterized the user of fitness tracking applications in three clusters regarding the perceived sensitivity.

Regarding the concerns, users o tracking appications are using these technologies but are also concerned. The majority agree that they don't have control over what will happen to their data or that it will be possible to create an exact profile of their movements, habits, or preferences. Interestingly, the research showed that former users are less concerned then users and none-users. The most investigated concerns are seen as neutral except for the fact that it will be possible to create an exact profile of their movements, habits, and/or preferences. Generally, this investigation stresses that there are concerns and data pieces which are characterized as sensitive. Nevertheless, the study showed that people are willing to use these technologies, even if they have concerns such as that app companies will misuse their data or that they have no control over what will happen to their data. In this case, there is the talk of the privacy paradox.

\section{References}

[1] Ilhan, A., \& Henkel, M. (2018). 10,000 Steps a Day for Health? User-based Evaluation of Wearable Activity Trackers. In Proceedings of the 51st Hawaii International Conference on System Sciences, January 3 - 6, 2018, Waikoloa Village (pp. 3376-3385). Honolulu, HI: HICSS (ScholarSpace).

[2] Feng, Y., \& Agosto, D.E. (early view). Revisiting personal information management through information practices with activity tracking technology. Journal of the Association for Information Science and Technology. https://doi.org/10.1002/asi.24253

[3] World Health Organization (WHO), Health Education and Promotion Unit, "The Healthpromoting workplace: making it happen". Geneva. http://www.who.int/iris/handle- /10665/64220, 1998. 